\title{
Syntely: Paradigm for an inductive psychology of memory, perception, and thinking
}

\author{
JOSEF PSOTKA \\ University of Waterloo, Waterloo, Ontario, Canada N2L $3 G 1$
}

\begin{abstract}
Two experiments investigated the effects of stimulus structure and human memory on human expectancy and concept formation. Sequences of binary events, $\mathrm{Xs}$ and Os, were given for inductive prediction and continuation under conditions of discrete (Experiment 1) and continuous (Experiment 2) presentation. The results of both experiments supported an interpretation that people base their predictions on a specific kind of structure found in binary sequences called syntely: the proportion of all earlier subsequences that match the end subsequences and also support the continuation of the terminal run of a binary sequence. An analysis of the concept formation process in terms of inductive cues and evidence is provided.
\end{abstract}

This paper provides a detailed data base and theoretical overview for an analysis of human thinking derived from the basic principle of thought, induction. Historically, thinking has been attacked experimentally within the framework of concept attainment, acquisition, reception, or selection (cf. Bourne, 1966; Bruner, Goodnow, \& Austin, 1956). Along a different path, continuing and extending the early work of Hull (1920), Posner and Keele (1970) have provided evidence that concepts or prototypes are developed through some kind of averaging process so that the prototype is derived as a central tendency of many different but similar representations. Others (Anglin, 1975, in press; Rosch, 1975) have determined that natural concepts cannot be completely described or defined by a definite number of attributes. Instead, concepts must be viewed as fuzzy, overlapping, or correlational. The formation of concepts is an inductive art. The analysis of induction presented in this paper accentuates the importance of averaging and correlational accretion in the formation of concepts.

\section{Binary Induction}

The binary prediction task has long been considered to demand the formation of inductions, inferences, or expectancies (Hake \& Hyman, 1953). The problem of induction is historically and philosophically venerable (Hume, 1961; Reichenbach, 1968; Russell, 1945) and topical (Swinburne, 1974). Binary sequences offer an

The main findings reported in this article were part of a dissertation submitted to Yale University in partial fulfillment of the requirements for a PhD degree. I especially thank Wendell Garner and Michael Kubovy for their encouragement and supervision. My thanks also go to Drs. R. P. Abelson, W. Kessen, and K. Nelson for their valuable comments and guidance. This research was supported by NIMH Grant MH 14229 to W. R. Garner at Yale University. Further support came from the author's Grant 037-7137 from the University of Waterloo. Requests for reprints should be sent to Josef Psotka, Department of Psychology, University of Waterloo, Waterloo, Ontario, Canada N2L 3G1. experimental and psychological, rather than philosophical, approach to the problem of induction.

An experiment by Psotka (1975) used all 70 binary sequences of Length 8 composed of four Xs and four Os. People were asked to read each sequence from left to right and predict the next element they thought should occur. People did not respond in a random fashion. There was considerable response variability from one sequence to another, but response proportions were highly reliable for any given sequence. A measure of serial patterns' structure called the syntely algorithm successfully predicted the response proportions continuing these serial patterns, where all previous models failed.

\section{Syntely Algorithm}

Syntely tries to describe something about the intrinsic structure of the external world: It is a stimulus measure like simplicity or similarity or symmetry. Syntely differs from these stimulus measures in the important respect that it is based on the principle of induction by enumeration. The syntely algorithm measures the degree to which the past evidence of the intrinsic structure of a binary pattern converges on a continuation or repetition of the last element of the pattern: It determines the degree to which a run response is appropriate. Given the binary sequence preceding a choice point, the algorithm finds all prior subsequences that are identical to the subsequences preceding the choice point, determines the proportion of those subsequences that are followed by runs, and predicts that people will choose a run in the same proportion. The algorithm also gives special emphasis to runs immediately preceding the choice point, as others have in the past (e.g., Royer \& Garner, 1966), by assuming that these runs are grouped into a unit on the basis of proximity and identity and are not broken in the search for prior matching subsequences unless nothing else is available. 
As an example, consider the binary sequence XOXXOOXO. The choice point is at the extreme right of the sequence. It is preceded by subsequences $O$, $\mathrm{XO}, \mathrm{OXO}, \mathrm{OOXO}$, etc.). Only the first three subsequences occur as patterns of runs and alternations in earlier parts of the sequence. The subsequence " $O$ " occurs seven times as a "pattern" of a single element (either an $\mathrm{X}$ or an $\mathrm{O}$ ). Only two of the seven patterns are followed by a run of the same element. The subsequence "XO" occurs four times as a pattern (either XO or OX) and two of these four patterns are followed by a run. The subsequence "OXO" occurs only once as the pattern "XOX" and it is followed by a run. Thus, $2+$ $2+1=5$ prior patterns out of 12 are followed by a run. The syntely of this sequence is therefore $5 / 12$, or .42 . This is the proportion of people who are expected to continue this sequence with the prediction of an "O."

\section{Syntely Model}

The algorithm may be interpreted in the light of experimental work on cued recall (Tulving \& Pearlstone, 1966). The terminal subsequences that are matched to the preceding patterns can be thought of as cues that reinvoke those patterns in a recall-like process (Anderson, 1973; Engel, Dougherty, \& Jones, 1973). The size of this terminal segment or cue is determined by the matching process, in the algorithm, to the preceding events which may be thought of as the evidence for the prediction. In this matching process, the cue and the evidence overlap to some extent, dependent on the length of the matched subsequences. This identification of a sequence as being composed of a cue and evidence in the context of a binary prediction task represents a conceptualization of the syntely algorithm's measure of the intrinsic structure of binary sequences which emphasizes the processes of memory in the formation of inductions.

This conceptualization identifies a process model of human induction. A stimulus cue reinstates past occurrences of events in some sense identical to the cue. The events in temporal contiguity with the recurrences are also reinstated. All of these events are averaged, composited, or accreted into a prototype or concept of the cue and of the expected event. The prototype of the cue can be stored as a pattern on which to base future inductive rules. The prototype of the expected event can be converted into a concrete response. To do this, it must be compared to concrete or specific events. A decision rule can determine which event the expected prototype is most like, based on a similarity measure using crosscorrelation like that proposed by Engel et al. (1973), Kabrisky (1964), Maher (1970), or van Heerden (1968). This event may then be predicted. If it is assumed that the decision rule uses a maximal likelihood criterion based on the size of the similarity measure, then this model makes the same predictions as the syntely algorithm when the noise distributions for the cued recall and averaging process, the similarity calculation, and the criterion placement assume appropriate variance and mean values.

\section{Marginal Frequency}

If there is an effect of marginal frequency that is independent of syntely, that effect may be the same for all members of the set of sequences with the same marginal frequency of the final event. The possible amount of this effect can be determined from the following analysis. The marginal frequency of the final event acts as one source of information. The mean syntely of the set acts as another source of information about the continuing structure of the set. There seems to be no obvious reason to treat either of these sources of information or structure as more important or influential than the other. So if these two sources of structure are combined to determine subjects' expectancies, a constant effect of subjects' run proportions for any particular sequence should be observed as an algebraic addition of the mean difference between the marginal frequency of the final event and the mean syntely of the set. In this way properties of the set of stimuli and properties of the individual sequences can combine to determine subjects' expectancies.

\section{EXPERIMENT 1}

The primary purpose of the experiment is to test the effect of intrinsic structure on run response proportions for sequences in which the marginal frequencies of binary events are unequal. This will be done first by showing that, for the 508 binary sequences varying in length from two to eight elements, the syntely measure correlates highly with subjects' run response proportions. Second, it will be shown that the relative importance of each position in determining subjects' predictions corresponds to the importance assigned by the syntely analysis.

\section{Method}

Subjects. Twenty-four subjects were recruited from an introductory psychology course and served in the experiment as part of a course requirement. Most of the subjects were freshmen or sophomores, and there were 16 males and 8 females.

Materials. The stimulus sequences were each of the 511 possible binary sequences varying in length from zero to eight events. They were presented individually, typed on $6.3 \times 8.8 \mathrm{~cm}$ cards with the top right-hand corner clipped, double spaced, in Coronamatic 2200 capital type centered on each card. The subject wrote his prediction for a sequence on the response sheet beside the number in the top left-hand corner of the card containing the sequence.

Procedure. The 24 subjects were tested individually. They were each seated at a table with the stimulus cards stacked in a file box and the response sheets lying in front of them. They were given the following instructions on a typewritten sheet: "This experiment is designed to find out how people make predictions about the ongoing course of events. It is not an experiment about ESP. You will be given a stack of 511 cards, with each card containing a short sequence of Os and Xs. Your 
task will be to read each sequence from left to right in the normal reading order to yourself and decide whether an $X$ or an $O$ would come next if the sequence continued. Of course, the sequence does not continue in this experiment, so there is no right or wrong. All the cards have been thoroughly shuffled, so treat each one independently of all the others. Each card has a number typed on it, and you are to write your choice on the response sheet beside this number. There are many sequences and you have only a little time, so do not worry too much about each sequence; just read it to yourself once and put down whatever you think should come next as quickly as you can. When you are finished return your work to the experimenter."

The subjects were then asked if they had any questions, and all questions were answered as completely as possible with restatements of the instructions. All subjects completed the task in less than $1 \mathrm{~h}$. The cards were thoroughly shuffled for each subject.

\section{Results and Discussion}

Means. The predictions of the 24 subjects were tabulated and the run response proportions for the 508 sequences were compared with the syntely measure for each sequence. The mean of the syntely measure was .322 , but that of the responses was .444 , a very large difference.

It was conjectured that this large difference between means was a result of the effect of marginal frequencies on subjects' predictions. To test this conjecture, scatter plots were drawn with subjects' run proportions as a function of the syntely of the sequences for each of the 28 sets of sequences defined by the marginal frequency of the final or rightmost element. An example of such a scatterplot is shown in Figure 1. As in this figure, when the marginal frequency of the final event was less than approximately .5 , the syntely measure consistently was larger than the subjects' run proportions for the sequences in the set, and the plotted points fell below the diagonal. It was also quite clear that when the marginal frequency of the final event was greater than approximately .5 the plotted points consistently fell above the diagonal. Chi-squared tests verified these observations at a very high level of statistical significance.

Correlations. In spite of the mean differences between the syntely measure and subjects' run proportions, the correlations between these two measures were significant for all sets of sequences of the same length with more than 10 members. The overall correlation between the syntely measure and subjects' run response proportions was .734 , indicating that the syntely measure did an effective job of describing subjects' responses for the 508 sequences used. Specifically, for the sets of sequences of Length 5,6, 7, and 8, the correlations between syntely and responses were .82 , $.78, .74$, and .71 , respectively.

Predicted adjustment. If the subjects take the marginal frequency of the final event and the syntely of the set of all sequences with the same marginal frequencies equally into account in making their predictions, it would be expected that the difference between the mean of their run proportions and the mean syntely of the set would equal one-half the difference between the margin- al frequency of the final event (MF) and the mean syntely of the set $(\bar{s}): 1 / 2(M F-\vec{s})$. To test this prediction, a scatterplot comparing the actual difference and the predicted adjustment was drawn in Figure 2. The

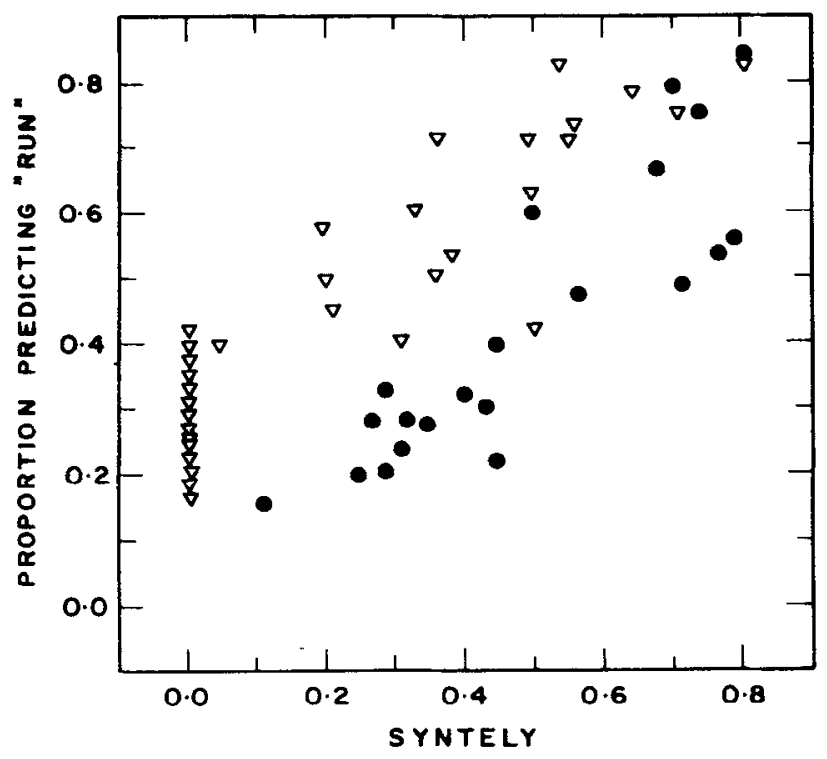

Figure 1. Subjects' run response proportions as a function of the measured syntely for two sets of sequences of the same length but with different marginal frequencies of the final event. The filled circles represent sequences in which the final event occurred three out of eight times. The open triangles represent sequences in which the final event occurred five out of eight times. Each point is the mean of a sequence and its complement, so it is based on 48 observations.

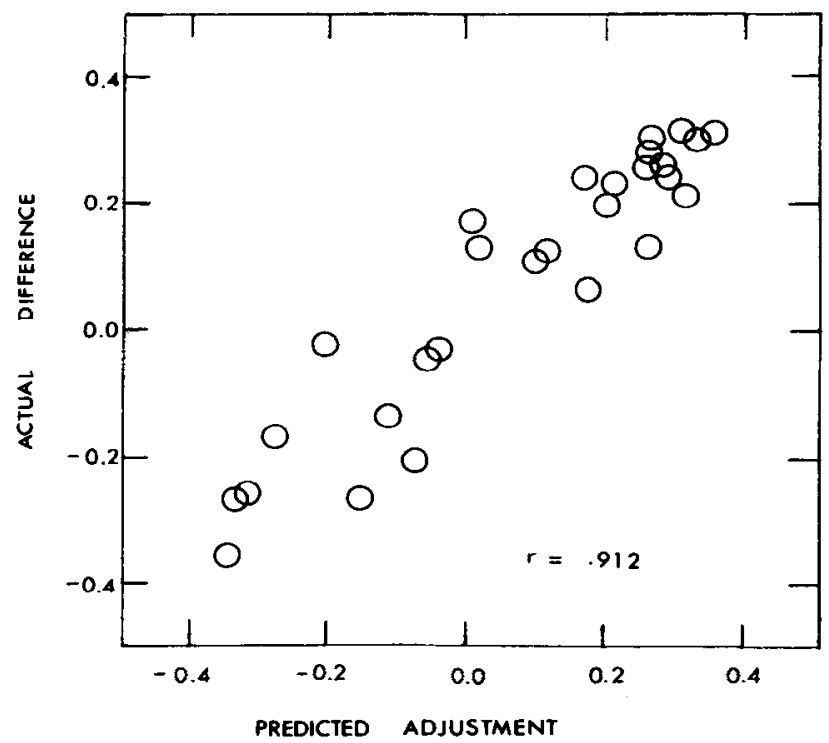

Figure 2. The predicted adjustment of the syntely measure in order to take marginal frequency into account, $1 / 2(\mathrm{MF}-\bar{s})$, as a function of the actual difference between the mean of the subjects' run proportions and the mean syntely for the 28 sets of different final event marginal frequencies extracted from the sequences of Experiment 1. 
correlation between these two measures of the effect of marginal frequency for the 28 different sets given in the discrete prediction data was .912 , and the least squares line fitting the data had a slope of unity and an intercept of zero. As may be seen in the scatterplot, the distribution of points along both axes was fairly homoscedastic.

One of the effects of subjects' sensitivity to marginal frequencies is to decrease the correlation between run responses and syntely over sets of sequences with different marginal frequencies. Adjusting the syntely measure for the effect of marginal frequency should increase this correlation. When this was done for the 508 sequences used, the overall correlation increased from .734 to .860 and the mean of the adjusted syntely measure became .448 , not significantly different from the mean of the subjects' run proportions of $\mathbf{4 4 4}$. The change in the overall correlation is significant $(\mathrm{p}<.05)$.

The importance of each position. The 256 sequences of Length 8 were sorted into nine sets of 128 paired sequences. One set was paired so that the member pairs were complements and treated as Position 0 . The other eight sets were paired so that the pairs matched at seven positions but were complementary at one position, for all eight possible positions. Within each set of pairs, this position was the same one. Treating the sequences in the same left-right order as subjects read them, these positions were numbered from one to eight. For each of

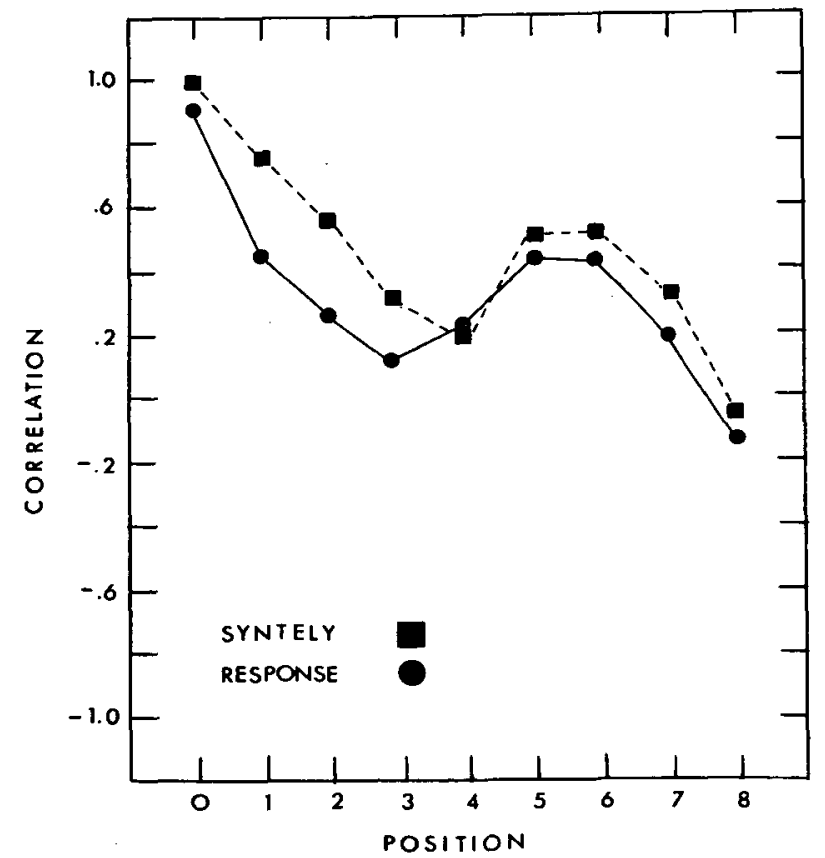

Figure 3. Intraclass correlations of sets of 128 paired sequences for 24 subjects' actual predictions, and for the adjusted syntely of the sequences. Nine sets of sequences were used, all with eight events, paired so that each pair differed only at the same single position numbered from left to right for each set. The correlations of complementary sequences (Position 0 ) are shown to provide a baseline and an index of noise in the predictions. the sets the run response proportions were correlated, and the adjusted syntely of the pairs was correlated. The correlations are plotted in Figure 3. In fact, the two functions are as expected and quite similar. Only Position 4 is slightly out of line: Subjects do not give it as much importance as the syntely analysis would suggest. This discrepancy may be the result of subjects' adoption of a prediction strategy that is specific to this length of sequence.

In order to counterbalance, or at least reduce, the effects of strategies associated with the length of a specific set of sequences, it is possible to correlate sequences of different lengths to demonstrate the importance of each position as it influences expectancy. The 256 sequences of Length 8 were paired with the 128 sequences of Length 7 to form eight sets of 256 pairs. Since each sequence of Length 7 is one event shorter than each sequence of Length 8 , the sequence of Length 7 may be treated as if it had a blank or no event at one of the eight positions of the sequences of Length 8 , where the positions are again numbered from left to right.

The correlations between the run responses for these pairs, and between the adjusted syntely for them, are shown for each position in Figure 4. The correlations for complementary sequences are again shown as Position 0 to provide an estimate of the reduction in correlation due to noise. The form of the two functions is almost

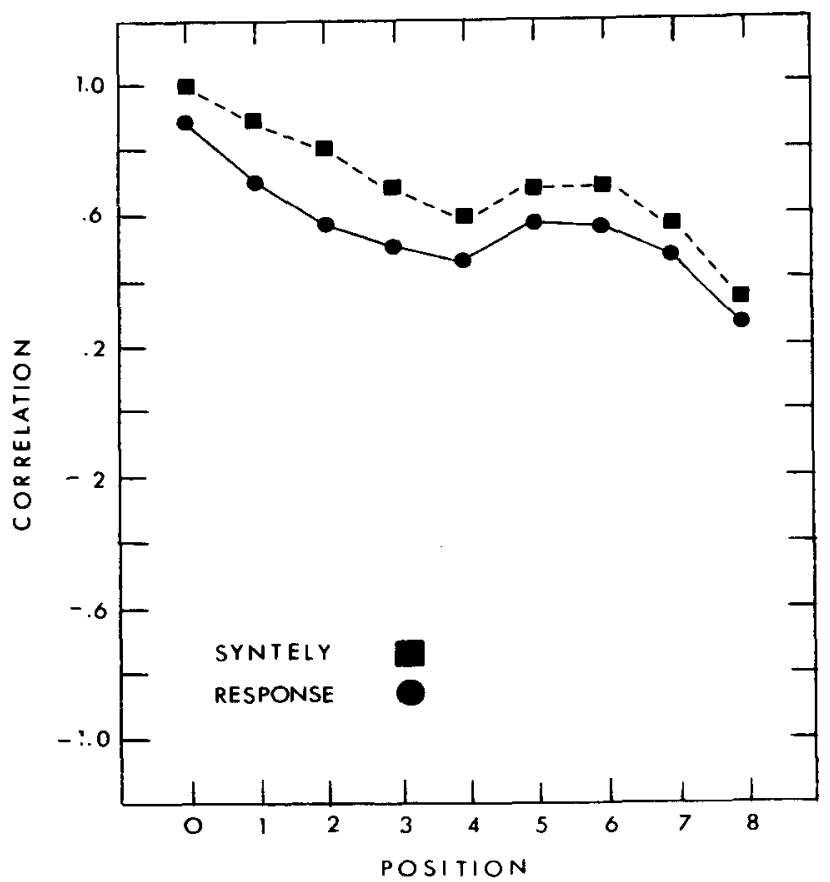

Figure 4. Product-moment correlations of sets of 256 sequences paired so that one member of a pair had eight events and the other had seven events. The sequences were identical, but the sequences of Length 7 were treated as if they had a blank at one of the eight positions of the corresponding pair member, numbered from left to right. The correlations of complementary sequences (Position 0) are shown to provide a baseline and an index of noise in the predictions. 
Table 1

Sequences Paired With XXXXXXXX

\begin{tabular}{ccc}
$\begin{array}{c}\text { Serial } \\
\text { Position }\end{array}$ & Autocorrelation & Crosscorrelation \\
\hline 0 & OOOOOOOO & OOOOOOOO \\
1 & OXXXXXXX & -XXXXXXX \\
2 & XOXXXXXX & X-XXXXXX \\
3 & XXOXXXXX & XX-XXXXX \\
4 & XXXOXXXX & XXX-XXXX \\
5 & XXXXOXXX & XXXX-XXX \\
6 & XXXXXOXX & XXXXX-XX \\
7 & XXXXXXOX & XXXXXX-X \\
8 & XXXXXXXO & XXXXXXX- \\
\hline
\end{tabular}

completely identical. The small discrepancy at Position 4 is eliminated. Since this analysis replaces each position with a blank rather than complementing it, the reduction in correlation at each position is generally only half the amount found in the previous analysis. Furthermore, the form of these two crosscorrelational functions is better in agreement with the syntely analysis autocorrelating sequences of Length 8. For instance, the inflection point is found at Position 4 in all three functions.

The same analyses were performed on sequences of Length 7 and on sequences of Length 6 . In general, the functions are of the same form and show the same relationships. Discrepancies between the syntely analysis and the subjects' responses found in the autocorrelational analysis of sequences of the same length are reduced when sequences of different length are crosscorrelated. Subjects seem to have specific strategies associated with a specific sequence length.

Perhaps this auto- and crosscorrelation analysis of serial position effects can be made more understandable with one complete example. Take the sequence

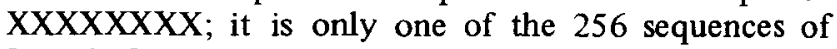
Length 8 . To determine the importance of each serial position, it would be paired with the sequences in Table 1. For each serial position 128 pairs of sequences can be formed. By correlating the response proportions, or the syntely measure, for these pairs of sequences, the importance of that position can be determined by the decrement in the size of the correlation relative to Serial Position 0.

\section{EXPERIMENT 2}

\section{Continuous Predictions}

Continuous predictions have commonly been used in the probability learning paradigm. A subject makes a prediction based on the previous history of a sequence and then is immediately told whether he is right or wrong by being given the next event in the sequence. Thus the major difference between a continuous prediction task and a discrete position task is the presence or absence of feedback provided by the sequence itself. Experiment 1 supplied excellent evidence that subjects are sensitive to the intrinsic structure of binary sequences and use this structure as a basis for their predictions in a discrete prediction task. The purpose of Experiment 2 was to try to determine whether subjects are sensitive to the intrinsic structure of binary sequences in a continuous prediction task.

\section{Cued Predictions}

The role of memory aids in the form of a display of the events immediately preceding a prediction has been the subject of considerable experimentation. Unfortunately, the main analytic tools have been speed of probability matching or degree of probability overshooting, and these tools have been unable to detect any effect of memory aids or cues (Wolin, Weichel, Terebinski, \& Hansford, 1965). The syntely analysis suggests that cues play an important role in human expectancy processing, and a display of immediately preceding events might allow subjects to use longer cues to reinvoke the prior evidence.

\section{Method}

Subjects. Seventy-two subjects were recruited from an introductory psychology course and served in the experiment as part of a course requirement. Most of the subjects were freshmen or sophomores and there were 57 males and 15 females.

Design. Six groups of 12 randomly assigned subjects participated in a continuous prediction task. In this task the subjects predicted the next event in one list of 264 elements as the list unfolded one element at a time. The six groups were defined by the factorial combination of two lists and three memory aid conditions where one, four, or eight events preceding the event to be predicted were in display before each prediction.

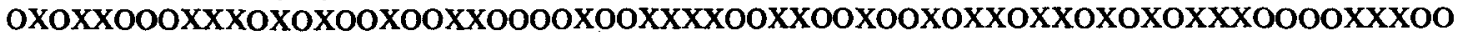
XXXoXXX00X0X0X0X0000X0XXXX0XX00000XXXXXX0XXXX00000000X0X00XX0X00X0

List 2

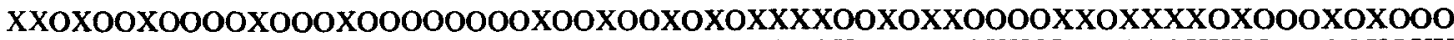
oOXX000000XXXoXX00XX000XX0X0000XXXX000X0XXX00XX0X0X000XXX00000X0XX oxox

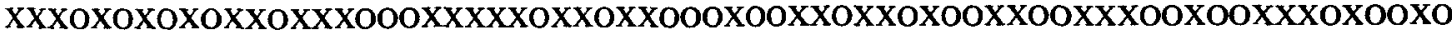


Materials. Two lists 264 binary elements in length were used. Each list contained all 256 binary sequences of Length 8 appearing once and overlapped throughout the list. In List 1, 244 of the sequences of Length 8 were followed by the complementary element to that occurring in List 2. The other 12 sequences of Length 8 were followed by the same element in both lists. Each list had 132 Xs and 132 Os. The lists are given in Table 2.

The lists were presented on $6.3 \times 8.8 \mathrm{~cm}$ cards numbered sequentially. The number of events on each card depended on the memory aid condition. When there was one memory aid, each card held only one event. When there were four memory aids, each card contained four events. When there were eight memory aids, each card contained eight events. In each of the six groups the first card contained the same sequence of eight events. The subject wrote his prediction on a response sheet headed "Prediction Responses." The six decks of continuous prediction list cards were, of course, presented in the same order to each subject. All decks were presented in a specially constructed metal file box that exposed only one end card at a time.

\section{Results and Discussion}

Subjects' run proportions were tallied for each memory aid condition within each list for all 256 sequences of Length 8 in each list. The mean run proportions for all six conditions were statistically greater than .5 , and greater than .44 , the run proportion in the discrete task of Experiment 1. Over all six conditions the mean run proportion was .548 , based on 18,432 observations. On the whole it may be induced that people dealt with the subsequences in this list predic-

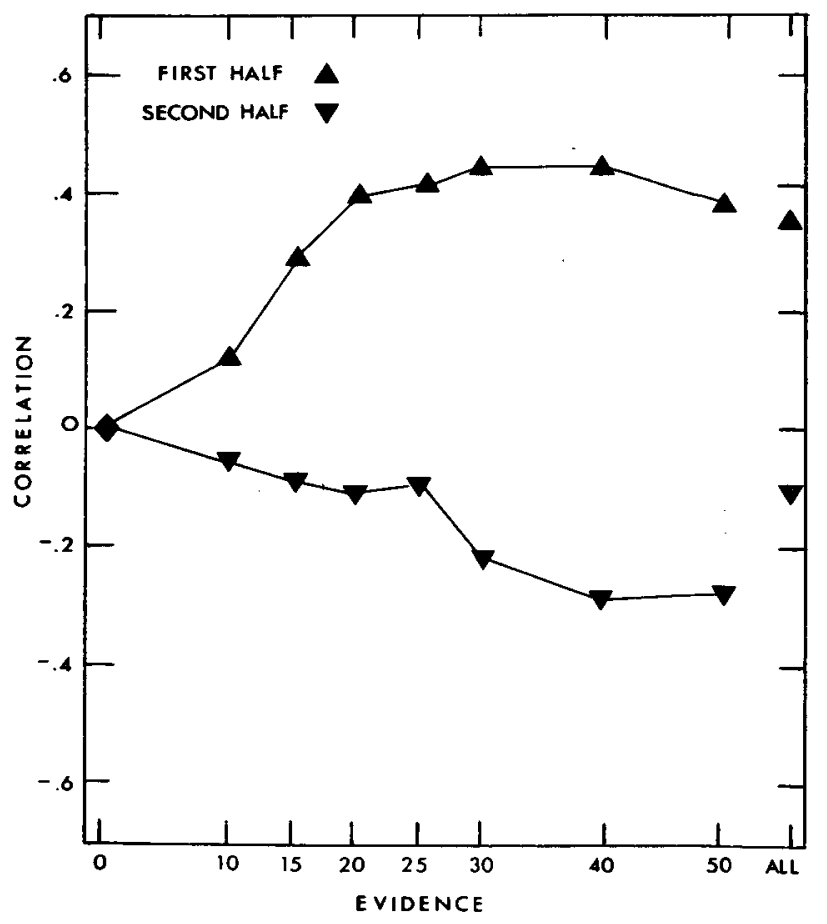

Figure 5. Correlations between the syntely measure and subjects' run response proportions for the first and second halves (128 events each) of List 1 as a function of the length of evidence used in syntely.

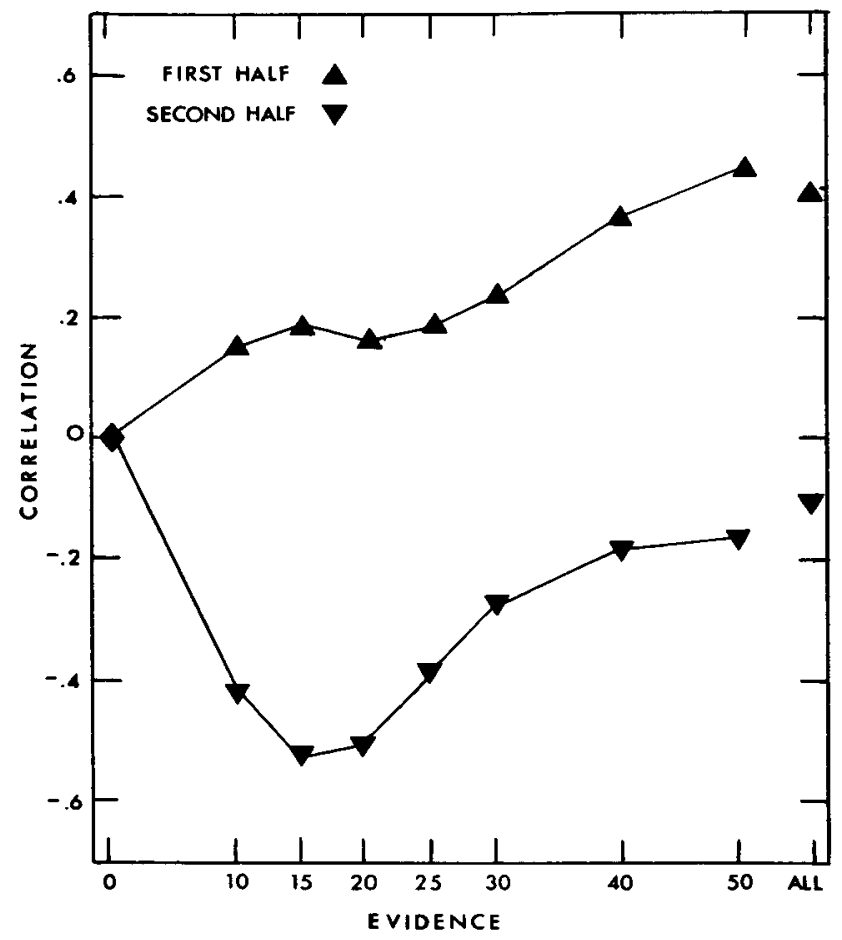

Figure 6. Correlations between the syntely measure and subjects' run response proportions for the first and second halves (128 events each) of List 2 as a function of the length of preceding evidence used in the syntely algorithm.

tion task in a different way than they did in the discrete prediction task of Experiment 1.

Correlations were also carried out for all six possible within-list comparisons of the run response proportions for each of the three memory aid conditions. All six within-list comparisons were significant: The mean correlation for List 1 was .218, and for List 2 the mean correlation was .355 . No significant differences were found among the three memory aid conditions in either list. Between-list correlations were not significant. These data seem to support the induction that people in this experiment were basing their predictions on the evidence of the structure of each list, using more than at least the eight preceding events prior to each prediction.

The amount of evidence used. The run responses were combined across memory aid conditions for each list and correlated with the syntely measure using the 10 , $15,20,25,30,40,50$, or all preceding events before each prediction as evidence in the syntely algorithms. These correlations were calculated for the first and second halves of each list to see if there were any changes over time with an increasing number of predictions. The results for List 1 are shown in Figure 5, and those for List 2 are shown in Figure 6.

The most striking characteristic of these two figures is the large change between correlations for the first and second halves of each list. For the first half of each 
list the correlations are consistently positive, and for the second half they are consistently negative. Both lists clearly demonstrate the same effect. The changeover from predicting events in accord with the syntely measure to predicting the complement would seem to stem from the fact that subjects are predicting a random sequence, so that on the whole, half their predictions are disconfirmed by the evidence of the next event. Given the situation of being wrong on so many occasions, it seems plausible that more and more subjects begin predicting the complementary event to the one suggested by the intrinsic structure of the sequence in order to take these consequences into account.

The second striking feature of these functions is the finding of maximum correlations using evidence considerably longer than would be expected form the results of direct recall tests. Although it is impossible with these data to specify the exact length of evidence for which the correlations are maximal, since there are likely to be subjects in both halves of the list predictions who predict in accord with the syntely and who predict the complement, it may be induced that most subjects were using at least the last 20 or 25 events preceding each prediction as evidence. All correlations greater than .175 in absolute value are significant $(\mathrm{p}<.05)$.

\section{GENERAL DISCUSSION}

\section{Implication for Concept Formation}

The prediction of the next event in these sequences is an inductive act, the solution of a problem, and the actualization of a concept. It is possible that this concept is formed neither by linguistic inference nor by whim, intuition, insight, or a particular conjunctive or disjunctive deductive strategy. The concept may be formed by a kind of average of the past evidence based on the appropriate context. In the formation of this average, no evidence is discarded except the evidence that does not fit the context of the terminal part of the sequence, or inductive cue. This kind of an averaging process model of concept formation is reminiscent of Galton's work on composite pictures (Galton, 1907; Semon, 1921; Psotka, Note 1).

\section{Implications for Memory}

The inductive analysis of serial position effects predicts that positive recency arises through the relation between recent and past portions of the time series of memory. The prediction task with all elements freely available for inspection provides little encouragement for rehearsal of individual elements in anything but their given order, so the rehearsal effects found with verbal materials to be recalled may safely be assumed not to exist: The positive recency recall curve is the appropriate one for comparison with the inductive syntely and response curves (Brodie, 1975; Rundus, 1971). The syntely analysis of inductive processes divides a to-be- predicted sequence into two distinct parts: the evidence, or past, part of the sequence and the inductive cue, or present, part. The demarcation between the evidence and the cue is determined by the relation of cue to past evidence. If the cue matches long parts of the evidence, then the cue will be long; otherwise, it will be relatively shorter. Given the random structure of cues and evidence found in the sequences used in these experiments, matches of every length are found with a frequency that is an inverse function of the length of the match: The result is positive recency. Most recall lists have a similar random structure of their own and so produce the same form of curve.

\section{Continuous Predictions and Memory}

The theoretical analysis proposed so far has placed no constraints on the limits of memory. The interpretation of the serial position effects for the syntely measure presupposes perfect memory of the contents of consciousness in order to produce the positive recency curve. But less than perfect memory would produce a very similar curve derived from response proportions, with algebraically lower correlations in the recency portion of the curve, indicating a greater relative importance of these positions. Although such differences were not observed in the discrete task, this is not surprising since the sequences were short and fully in display. However, the processing was done mentally, so memory was involved. Perhaps the only limitation on the accuracy of memory as detected by a performance measure is the adequacy of the inductive cue to create the appropriate concept that reveals the presence of memory.

The syntely analysis has indicated that subjects use much longer sequences of evidence as a basis for predictions than would have been thought possible on the basis of direct recall tests. Unfortunately, because of the change in subjects' prediction strategy from the beginning to the end of long, continuously predicted sequences, the exact length of this evidence was not possible to establish. Since it is likely that this strategy shift occurred because of the feedback provided in a continuous prediction task, it would seem reasonable to predict that this use of evidence may be better analyzed by using long sequences of events with only one element in display at a time, but requiring only one discrete prediction at the end.

\section{REFERENCE NOTE}

1. Psotka, J. On predicting binary sequences: Memory, induction, and abstraction. Paper presented at the 37 th annual meeting of the Canadian Psychological Association, Toronto, Ontario, June 1976.

\section{REFERENCES}

ANDERSon, J. A. A theory for the recognition of items from short memorized lists. Psychological Review, 1973, 80, 417-438. 
ANGLrn, J. M. The child's first terms of reference. In S. Ehrlich \& E. Tulving (Eds.), Bulletin de Psychologie. Special issue on semantic memory, 1975.

ANGLIN, J. M. Word, object, and conceptual development. New York: Norton, in press.

BoURNe, L. Human conceptual behavior. Boston: Allyn \& Bacon, 1966.

Brodre, D. A. Free recall measures of short-term store: Are rehearsal and order of recall data necessary? Memory \& Cognition, 1975, 3, 653-662.

Bruner, J. S., Goodnow, J. J., \& Austin, G. A. A study of thinking. New York: Wiley, 1956.

Engel, G. R., Dougherty, W., \& Jones, B. R. Autocorrelation and the confusibility of the alphabet. Canadian Journal of Psychology, 1973, 27, 317-326.

Galton, Sir F. Inquiries into human faculty and its development. London: Dent, 1907.

Hake, H. W., \& Hrman, R. Perception of the statistical structure of a random series of binary symbols. Journal of Experimental Psychology, 1953, 45, 64-74.

HuLl, C. L. Quantitative aspects of the evolution of concepts. Psychological Monographs, 1920, 28(1, Whole No. 123).

Hume, D. A treatise of human nature. London: Dent, 1961.

KABRISKY, M. A proposed model for visual information processing in the human brain. Urbana: University of Illinois Press, 1964.

MAHER, F. A. A correlation of human and machine pattern discrimination. Institute of Electronic Engineers Naecon Record, 1970, 46, 260-264.

Posner, M. I., \& Keele, S. W. Retention of abstract ideas. Journal of Experimental Psychology, 1970, 83, 304-308.
Psotka, J. Simplicity, symmetry, and syntely: Stimulus measures of binary pattern structure. Memory \& Cognition, 1975, 3, 434-444. REICHENBACH, H. The rise of scientific philosophy. Berkeley: University of California Press, 1968.

Rosch, E. H. Classifications of real-world objects: Origins and representations in cognition. In S. Ehrlich \& E. Tulving (Eds.), Bulletin de Psychologie. Special issue on semantic memory, 1975.

ROYER, F. L., \& GARNER, W. R. Response uncertainty and perceptual difficulty of auditory temporal patterns. Perception \& Psychophysics, 1966, 1, 41.47.

RunDus, D. Analysis of rehearsal processes in free-recall. Journal of Experimental Psychology, 1971, 89, 63-77.

Russell, B. A history of western philosophy. New York: Simon \& Schuster, 1945.

SEMon, R. The mneme. L. Simon (Trans.). London: Allen \& Unwin, 1921.

SWINBurne, R. (Ed.). The justification of induction. Toronto: Oxford University Press, 1974

Tulving, E., \& Pearlstone, Z. Availability versus accessibility of information in memory for words. Joumal of Verbal Learning and Verbal Behavior, 1966, 5, 381-391.

VAN HeERDEN, P. J. The foundation of empirical knowledge. Wassenaar: N. V. Uitgeverij Wistik, 1968.

Wolin, B. R., Weichel, R., Terebinsk, S. J., \& Hansford, E. A. Performance on complexly patterned binary event sequences. Psychological Monographs, 1965, 79(7, Whole No. 600).

(Received for publication February 23, 1977; accepted May 20, 1977.) 